\title{
Improving the safety of remote site emergency airway management
}

\author{
Julian Wijesuriya, Jonathan Brand \\ Darlington Memorial Hospital, County Durham, UK
}

\begin{abstract}
Airway management, particularly in non-theatre settings, is an area of anaesthesia and critical care associated with significant risk of morbidity \& mortality, as highlighted during the 4th National Audit Project of the Royal College of Anaesthetists (NAP4). A survey of junior anaesthetists at our hospital highlighted a lack of confidence and perceived lack of safety in emergency airway management, especially in non-theatre settings.

We developed and implemented a multifaceted airway package designed to improve the safety of remote site airway management. A Rapid Sequence Induction (RSI) checklist was developed; this was combined with new advanced airway equipment and drugs bags. Additionally, new carbon dioxide detector filters were procured in order to comply with NAP4 monitoring recommendations.

The RSI checklists were placed in key locations throughout the hospital and the drugs and advanced airway equipment bags were centralised in the Intensive Care Unit (ICU). It was agreed with the senior nursing staff that an appropriately trained ICU nurse would attend all emergency situations with new airway resources upon request. Departmental guidelines were updated to include details of the new resources and the oncall anaesthetist's responsibilities regarding checks and maintenance.
\end{abstract}

Following our intervention trainees reported higher confidence levels regarding remote site emergency airway management. Nine trusts within the Northern Region were surveyed and we found large variations in the provision of remote site airway management resources.

Complications in remote site airway management due lack of available appropriate drugs, equipment or trained staff are potentially life threatening and completely avoidable. Utilising the intervention package an anaesthetist would be able to safely plan and prepare for airway management in any setting. They would subsequently have the drugs, equipment, and trained assistance required to manage any difficulties or complications. We suggest that this should be the gold standard of airway resource provision and is in line with NAP4 recommendations.

\section{Problem}

Airway management is an aspect of anaesthesia and critical care that can be associated with considerable risk of morbidity and mortality (1). Reports from junior anaesthetists at our district general hospital highlighted a lack of confidence and perceived lack of safety in emergency airway management, especially in the nontheatre setting.

\section{Background}

Airway management is an important but potentially hazardous area of anaesthesia and critical care and was the subject of investigation during the 4th National Audit Project of the Royal College of Anaesthetists (NAP4) (2). The authors studied airway complications occurring in both the theatre and remote site environments. Remote sites are considered to be any location outside of the main theatre suite and where assistance from another anaesthetist is not always readily available (3).

NAP4 highlighted that endotracheal intubation of critically ill patients, in particular outside of the theatre setting, was associated with a higher risk of morbidity and mortality with common complications including; hypoxaemia, cardiovascular collapse, aspiration, oesophageal intubation, and death. The study also demonstrated that $61 \%$ of intensive care unit (ICU) patients experiencing an airway-related incident suffered neurological injury or death, compared with $14 \%$ of patients in the theatre environment. The reasons for this were plentiful, however; lack of equipment, lack of operator experience, lack of appropriate planning, and poor communication were deemed to be of significant importance in causing patient harm (2).

Human factors (or non-technical skills) are therefore of great importance in remote site emergency airway management and are defined as 'cognitive and social skills that reduce human error, improve human performance and enhance safety' (4). Nontechnical skills have been a recent area of study in both anaesthesia and intensive care medicine, and of particular importance is the non-technical skill of situational awareness. That is, an ability to maintain an overall awareness of the environment and anticipate potential problems, acting to prevent them from occurring. Numerous studies have revealed that a loss of situational awareness is often a key deficiency in many critical incidents leading to harm (5).

A key recommendation from NAP4 was that an intubation checklist is used alongside capnography in all remote site emergency airway management situations involving critically ill patients, as they have 
been shown to significantly reduce complication rates (2). Checklists are not a new concept and have been successfully used by the aviation industry for a long time. They are also widely used in the pre-hospital and military environments to improve patient safety in high-risk situations. The aim of using a checklist in clinical practice is to ensure that patient, equipment, and team-related factors are all addressed prior to the procedure and that the principles of crew resource management are used to improve the situational awareness of the team as a whole (4). The Resuscitation Council UK have recently published a minimum equipment and drug list (6) in an attempt to standardise drug and equipment availability for cardiopulmonary resuscitation. This list includes advanced airway equipment (including capnography) alongside core resuscitation drugs that when present will improve both safety and care delivery to patients. In addition, studies have demonstrated that intubation checklists and care bundles when used in critical care improve patient safety, reduce mortality, reduce complication rates, and have no effect on the time to induction, irrespective of anaesthetist grade $(7,8)$ therefore further supporting the NAP4 recommendations (2).

In the UK, training in intensive care medicine is changing, and doctors from differing backgrounds, who may have less advanced airway experience compared to their predecessors, may staff ICUs. This therefore means that remote site emergency airway management is of paramount importance in the maintenance of patient safety and steps need to be established to minimise the potential for patient harm occurring as a result of incidents relating to emergency airway management.

\section{Baseline Measurement}

The nature of the issue being assessed required a multi-faceted approach to investigation. An initial assessment of the available resources for remote site emergency airway management was made in all relevant clinical areas with the conclusion that available resources varied depending on the clinical setting.

In the Accident \& Emergency (A\&E) department, airway equipment and anaesthetic drugs were available at each resuscitation bay and a difficult airway trolley was available. This was applicable for both adult and paediatric resuscitation areas. However, stock levels of this equipment were not regularly checked, therefore leading to inadequate stock in addition to a marked variation in the experience and confidence of staff when using such equipment.

In ICU, both airway and difficult airway trolleys were available alongside all required drugs. All ICU staff were trained and experienced in assisting with advanced airway management. The trolleys and drugs were also inventoried and checked on a daily basis.

In the ward setting, there was no advanced airway equipment available and there were no anaesthetic drugs available. The significant majority of staff were also unfamiliar with assisting with airway management.

Subsequently, anaesthetic trainees who were 1st on-call and therefore primary responders to emergency situations were surveyed. They were asked about their confidence in providing emergency airway management and performing rapid sequence inductions (RSl's) inside and outside of the theatre environment with a questionnaire using Likert scales. The results demonstrated a significant lack of confidence across all trainees in remote site emergency airway management as compared to the theatre environment (Table 1).

\section{Design}

Following the survey and assessment of resources a number of interventions were made in order to improve the safety of remote site emergency airway management and to increase the confidence of anaesthetic trainees.

Firstly, an RSI checklist (Annex A) was developed for use prior to any $\mathrm{RSI}$ in order to ensure adequate communication, team composition, patient / equipment preparation, and planning for the management of anticipated difficulty or complications.

Two pre-existing drug bags were stocked to include all emergency anaesthetic and peri-arrest and cardiac arrest drugs. Appropriately sized syringes, needles, and drug labels were also included in a user-friendly and uniform layout. A photographic inventory with drug list was also attached to each bag to help in re-stocking after each use (Annex B).

An advanced airway bag was developed to include all equipment required for emergency, difficult, and failed intubations. It included the equipment required to perform all stages of the Difficult Airway Society (DAS) failed intubation guidelines (9). An inventory was also composed and attached to the bag to help in re-stocking after each use (Annex C).

As per NAP4 guidance (2), capnography is fundamental to aid confirmation and monitoring of endotracheal intubation regardless of the site of intubation, therefore, carbon dioxide detector filters were procured (following discussion with and approval from the Clinical Director) and these formed part of the airway equipment bag.

Each part of the above package was subjected to open consultation from all anaesthetic consultants and trainees, theatre sisters and staff, ICU sisters and staff, outreach team members, A\&E consultants, and resuscitation officers. A number of modifications were undertaken from the draft versions based on valid suggestions occurring during the consultation period.

\section{Strategy}

The RSI checklists were printed, laminated, and placed in key locations throughout the hospital. They were placed on the walls in both adult and paediatric resuscitation areas in poster format. In A4 size, they were attached to adult and paediatric resuscitation bay airway trolleys, the difficult intubation trolleys in A\&E, ICU and main theatre, and to the new advanced airway equipment bag. 
The drugs and advanced airway equipment bags were placed in the ICU pharmacy (drugs bags in fridge), with posters advertising their location. Following discussion at the ICU senior sisters meeting, it was agreed that upon request by the on-call anaesthetist, an appropriately trained ICU nurse would attend emergency situations with the RSI checklist, advanced airway equipment and drugs bags.

The departmental guidelines were updated to include a section providing information about the RSI checklist, drugs bag, advanced airway equipment bag, and availability of assistance. The 1st oncall anaesthetist's roles and responsibilities were also updated to include checking of the drugs and advanced airway equipment bags against their respective inventories at the start of each on-call shift, and replenishing stock if any deficiencies were found and after any clinical use. The use of zip ties on the zips of all bags was also introduced to prevent inadvertent use of the bag contents in nonemergency situations and also to alert users that the bag had been opened and used.

\section{Results}

Similarly to the baseline measurement, the effects of the intervention were difficult to quantify. Anaesthetic trainees informally reported higher confidence levels following the interventions, and the project was well received by the all departments commonly involved in remote site emergency airway management.

For interest, a telephone survey of nine trusts in the Northern Deanery was performed to assess the level of provision for remote site emergency airway management across the Deanery and large variations were found. From the nine Trusts contacted eight (89\%) had a designated advanced airway bag, but only one (11\%), included difficult / failed intubation equipment and similarly, only one $(11 \%)$, routinely used an RSI checklist for non-theatre airway management. In six trusts (66\%) a drugs bag was available for emergency non-theatre anaesthesia.

Further formal survey of anaesthetic trainees within the trust is planned to quantify the improvement in confidence levels, as well as an audit of drugs / equipment checking. This will be performed again using a questionnaire-based survey and will look at confidence levels with remote site airway management following the implementation of our package and will be undertaken on an annual basis. It is planned that an anaesthetic trainee be allocated to this role each training year in order to monitor, maintain and develop the safety improvement package.

The RSI checklist and emergency drug and airway bags are also to be used during a new educational programme aiming to increase the knowledge, confidence, and experience of A\&E staff in assisting with remote site emergency airway management and the management of its potential complications. The programme is planned to be multidisciplinary in nature involving those likely to undertake an RSI in the emergency department. Principally this means: junior anaesthetists, A\&E staff nurses, anaesthetic practitioners, outreach team members, and ICU staff nurses. The programme will initially consist of lectures that discuss remote site emergency airway management and its inherent dangers and how they can best be avoided. The concept of our package and its contents will also be introduced by way of a workshop where all candidates can familiarise themselves with the contents of our bags and with any unfamiliar airway equipment. Clinical simulation will then be used to allow multidisciplinary teams to undertake clinical scenarios based around remote site emergency airway management problems using both the RSI checklist and emergency bags we have introduced. The teaching days will be for limited numbers to ensure maximal educational benefit for those attending and will continue until all required staff have attended and are confident with the use of our airway package.

See supplementary file: ds2449.doc - "Supplementary Material Annexes (A, B, C)"

\section{Lessons and Limitations}

Firstly, it is our experience during this project that all staff, no matter what their role, are keen to adopt strategies aimed to enhance patient safety and increase cohesive inter-departmental working.

This project highlighted to us the importance of seeking repeated discussions with other clinicians and healthcare staff prior to implementation of our package. We circulated the proposed package in draft form to a 'review body' of key staff that included; anaesthetic consultants and trainees, theatre sisters and staff, ICU sisters and staff, outreach team members, A\&E consultants, and resuscitation officers for both comments and suggestions prior to implementation. Their opinions and suggestions were crucial in enhancing the success of the project as they highlighted unforeseen problems and subtle changes that have improved the success of the project. This also had the added advantage that it allowed familiarity with the project so that it was not new to staff when it was finally introduced into the clinical environment.

Regular discussion with the Clinical Director of Anaesthesia provided the experience and advice necessary for smoothly implementing a package such as ours and helped tailor the project to fit with the nuances of the individual hospital. The Clinical Director was also able to authorise reallocation of equipment resources, changes to the departmental guidelines, and use of departmental finances to procure new equipment. Of particular importance were the discussions with the ICU sisters and nursing staff and outreach team members. Discussion of the project at the ICU senior sister's meetings highlighted issues of logistics and responsibility with regard to the storage, checking and maintenance of the drugs and equipment, which were successfully resolved. The same meetings lead to the offer of a trained ICU nurse to accompany the anaesthetist and airway package to the situations involving remote site airway management in order to provide skilled assistance.

We would therefore fully support the opinions of others prior to embarking on any other clinical improvement project to ensure that the best work is produced.

This project unfortunately did have some limitations. Firstly, this project was limited to a single centre implementation. This means 
that despite its general applicability to other centres, the model used in this project may need adapting dependent upon the resources available in different centres. Potential examples may include in larger hospitals having more that one ICU or in smaller centres, with limited staff availability, it may not be possible to have immediately available skilled staff to help the anaesthetist with remote site airway management.

The numbers involved in the assessment of trainee confidence in this study are small and therefore to increase validity, this should ideally be repeated at a Deanery level. However, despite the small numbers, we do feel that the sample does represent the majority of most junior trainees when faced with such situations.

A potential problem that may arise with the introduction of this package is the encouragement of more senior staff in using the RSI checklist. We did not have this problem in our cohort of senior anaesthetists; however, it could be envisaged that senior staff may feel less inclined to use such a checklist due to their wealth of experience and skill. Unfortunately, complications and problems do happen to clinicians regardless of seniority, and it should be stressed that this package is an aid to simply enhance patient safety by ensuring any potential difficulties and / or complications are planned for as best as possible prior to commencing what is deemed a high-risk procedure (1).

There is always inherent difficulty when quantifying the difficulties with remote site airway management, particularly if difficulties occur but no harm comes to the patient as a result. However, the introduction of our airway package will allow for assessment of the use of the checklist and also whether essential help and equipment are present during such situations, and early reports are suggesting that it is already helping to promote good team working, identification of team roles and in promoting an environment where potential difficulties are actively planned for and avoided.

\section{Conclusion}

Delays or complications in remote site airway management due to lack of available appropriate drugs, equipment, or trained staff are potentially life threatening and completely avoidable.

As non-theatre emergency intubations are among the most difficult and are associated with the highest risk, it is intuitive that safetychecks should take place prior to RSI and difficult / failed intubation equipment should be immediately available along with appropriately trained staff. In such situations there is often insufficient time to obtain specific or specialist life-saving equipment meaning that the above preparations, if undertaken, are often lifesaving.

The vision in designing this project was to develop a relatively small but effective intervention package encompassing all the required resources to improve the safety and reduce the complications associated with remote site anaesthesia. Utilising our intervention package an anaesthetist would be able to safely plan and prepare for emergency airway management in any setting. They would subsequently have the drugs, equipment, and trained assistance required to safely manage any difficulty or complication without delay (including plans A-D of the DAS protocol) (9).

Though difficult to quantify, the body of evidence from NAP4 would imply that any steps taken to reduce the risk associated with airway management, in particular relation to high-risk patient groups and the clinical settings described previously, would only enhance patient safety.

Despite anecdotally finding increased confidence levels amongst anaesthetic staff following our intervention, we were surprised at the variation in provisions for remote site airway management between different hospitals in our Deanery in light of the NAP4 recommendations (2). We would therefore suggest that the intervention package developed in this project be the gold standard of resource provision for increasing the safety of remote site emergency airway management.

\section{References}

1. Simpson, G.D., Ross, M.J., McKeown, D.W. \& Ray, D.C. Tracheal intubation in the critically ill: a multi-centre national study of practice and complications. British Journal of Anaesthesia 2012; 108: 792-799

2. Cook, T.M., Woodall, N., Harper, J. \& Benger, J. Major complications of airway management in the UK; results of the Fourth National Audit Project of the Royal College of Anaesthetists. British Journal of Anaesthesia 2011; 106: 632-642

3. Royal College of Anaesthetists. Anaesthetic Services in Remote Sites. RCoA Guidance, 2011

4. Flin, R., Goeters, K. \& Amalberti, R. The development of the NOTECHS system for evaluating pilots' CRM skills. Human factors and Aerospace Safety 2003; 3: 95-117

5. Flin, R., O'Connor, P. \& Crichton, M. Safety at the Sharp End. A Guide to Non-Technical Skills. Ashgate: Aldershot, 2008

6. Resuscitation Council (UK). Minimum equipment and drug lists for cardiopulmonary resuscitation: Acute Care. Resuscitation Council (UK), 2013

7. Babolhavaeji, F., Rees, I., Maloney, J., Walker, J. \& Knights, $M$. Checklist for emergency induction of anaesthesia in critical care. Anaesthesia 2013; 68: 661

8. Jaber, S., Jung, B., Corne, P., Sebbane, M., Muller, L., Chanques, G., Verzilli, D., Jonquet, O., Eledjam, J.J. \& Lefrant, J.Y. An intervention to decrease complications related to endotracheal intubation in the intensive care unit: a prospective, multiple-centre study. Intensive Care Medicine 2010; 36: 248 - 255

9. Henderson, J.J., Popat, M.T., Latto, I.P. \& Pearce, A.C. Difficult Airway Society guidelines for the management of the unanticipated difficult intubation. Anaesthesia 2004; 59: 675-694

\section{Declaration of interests}

Nothing to declare. 


\section{Acknowledgements}

Anaesthetic trainees and nursing staff, Darlington Memorial Hospital.

Baskar Manickam (Clinical Director), Anaesthetic Department, Darlington Memorial Hospital 